\title{
On the role of the microporous layer in PEMFC operation
}

Jeff T. Gostick, Marios A. Ioannidis*, Michael W. Fowler and Mark D. Pritzker

Department of Chemical Engineering, University of Waterloo, Waterloo, ON N2L 5N5

*Corresponding Author: mioannid@uwaterloo.ca 


\section{Abstract}

The condition of liquid water breakthrough at the cathode of polymer electrolyte fuel cells (PEMFC) is studied experimentally and data on corresponding water saturation and capillary pressure are provided for gas diffusion layers (GDL) with and without a microporous layer (MPL). The data demonstrate that the GDL saturation at water breakthrough is drastically reduced from ca. $25 \%$ to ca. $5 \%$ in the presence of MPL. This observation is consistent with considerations of invasion percolation in finite-size lattices and suggests an explanation for the role of MPL in improving PEMFC performance at high current densities.

Keywords: polymer electrolyte membrane fuel cell, gas diffusion layer, microporous layer, water management, capillary pressure, percolation 


\section{Introduction}

The accumulation of excessive product water in polymer electrolyte membrane fuel cells (PEMFC) is a factor limiting performance at high current densities. Liquid water generated by the cathode reaction percolates through the porous gas diffusion layer (GDL) towards the gas channel. Notably, the capillary number and viscosity ratio prevailing during displacement of the gaseous phase by water are such that a highly ramified, finger-like pattern characteristic of invasion percolation is established [1,2]. Consequently, water saturation in the GDL and resistance to gaseous reactant transport both increase until water first breaks through at the GDL-channel interface. The liquid saturation and capillary pressure associated with water breakthrough in GDL materials are clearly important for understanding resistance to gaseous reactant transport due to water accumulation.

Efforts to improve PEMFC water management have focused on (1) impregnating the GDL pores with a hydrophobic polymer and (2) adding a micro-porous layer (MPL) on one face of the GDL. A recent systematic experimental study of these treatments by Ramasamy et al. [3] has clearly demonstrated improved mass transfer when these treatments are applied, both in terms of increased limiting current as well as reduced mass transfer resistance measured via AC impedance. The MPL treatment has been shown to be particularly beneficial although the actual function of this layer is still under debate. The MPL presumably creates better electrical and thermal contact between the catalyst layer and the GDL by providing a smoother, more continuous interface, but the benefits of the MPL are most noticeable at higher current conditions indicating that it somehow improves mass transfer. Since the MPL actually adds a diffusive resistance to mass transfer, it is generally thought that the MPL somehow alters the liquid water distribution in the cell to a more favorable arrangement for 
gas phase transport $[4,5]$. The MPL question has been recently addressed with full-cell models $[6,7]$, which predict that the MPL acts as a capillary barrier to water entering the cathode GDL and forces water to permeate from the cathode to the anode. Experimental attempts to confirm this mechanism have been inconclusive or contradictory. Using a transparent flow field to monitor water in the anode, Spernjak et al. [8] found that water appears in the anode channels only when an MPL is used on the cathode side, which they offer as proof for the function of MPL as a capillary barrier. Similar experiments by Ge and Wang [9], however, have attributed the appearance of water in the anode to condensation since water droplets only appeared on channel walls and were never observed emerging from the GDL. Atiyeh et al. [10] conducted quantitative water balance experiments and found no discernible increase in water collected from the anode when an MPL is present on the cathode for any given set of operating conditions.

In this work ex-situ measurements are presented of the water saturation and associated capillary pressure precisely at the point of water breakthrough in GDL samples with and without MPL. It is demonstrated that the water saturation at breakthrough is dominated by finite-size effects owing to the very small thickness of the GDL. Namely, when the entire inlet face of the GDL is exposed to water (i.e. no MPL), a significant fraction of the pore volume is invaded by non-percolating clusters of pores filled with water and $S_{w}^{\circ} \approx 0.27$ at breakthrough in the sample tested. When access of water to the inlet face of the GDL is restricted to a very small area, the number of non-percolating clusters of water-filled pores present at breakthrough [2] is drastically reduced and $S_{w}^{\circ} \approx 0.05$. In the presence of MPL, $S_{w}^{o} \approx 0.03$ at breakthrough, suggesting that the MPL restricts the number of points of entry of water into the GDL. 


\section{Experimental}

The simultaneous measurement of capillary pressure and water saturation at breakthrough was achieved with a simple modification of a technique detailed elsewhere [11]. In order to determine the point on the capillary pressure curve where breakthrough occurred, a small circular piece $(1 \mathrm{~cm}$ diameter) of dry hydrophilic membrane was inserted above the GDL sample and below the hydrophobic capillary barrier. The test proceeded as normal, with each capillary pressure change resulting in a saturation change up to the point of water breakthrough. The breakthrough point can be easily detected since the saturation changes drastically and rapidly due to wicking of water into the dry hydrophilic membrane placed above the sample. Figure 1(left) shows the water uptake response obtained from this test. The point of water breakthrough is clearly visible as a sudden change in GDL water uptake, allowing the saturation and capillary pressure just before breakthrough can be easily determined. Figure 1(right) shows that the capillary pressure curve up to the point of breakthrough obtained with the modified method is essentially the same as the capillary pressure curve obtained for the same material with the unmodified method [11]. Slight differences in the two curves are due to the fact that they were obtained from different samples.

To independently verify the breakthrough points found using the modified capillary pressure device, a second test was performed similar to that of Benziger et al. [12]. A piece of GDL was affixed to the end of a tube and the head of water above the sample was increased in 5 $\mathrm{cm}$ increments, waiting 5 minutes at each point, until breakthrough was observed. This simple test does not provide saturation at breakthrough, but confirms that the modified capillary pressure device observes the correct breakthrough pressure. 
Tests were performed on SGL10BA and SGL10BB gas diffusion layers. These materials had the same GDL substrate and hydrophobic polymer content (5 wt $\%$ ). The SGL10BB sample had an 80-100 $\mu \mathrm{m}$ thick MPL applied on one side [13]. In tests with the SGL10BB sample, the MPL was placed facing the water inlet as well as away from it. These tests are referred to as SGL10BB-I and SGL10BB-O, respectively. Tests were also conducted on an SGL10BA sample where the inlet face of the sample is covered with an impermeable PTFE mask having a $600-\mu \mathrm{m}$ hole punctured in the center for water flow (denoted SGL10BA-P). Tests on SGL10BA with the full face exposed to liquid water are denoted as SGL10BA-F. 


\section{Results and Discussion}

The main findings of this study are summarized in Figure 2. Regarding the plain GDL substrate material (SGL10BA), it is evident that the $P_{c}-S_{w}$ relationship for water injection is significantly affected by finite-size effects. Since GDL materials are very much thinner (ca. 10-20 pores across) than they are wide [14], a significant fraction of the sample pore volume is invaded by non-percolating clusters before breakthrough if the entire inlet face of the sample is exposed to water (SGL10BA-F). In this case, water breakthrough occurs at $P_{c}^{o} \approx 2500 \mathrm{~Pa}$ and a high saturation $\left(S_{w}^{o} \approx 0.27\right)$. By comparison $P_{c}^{o} \approx 3800 \mathrm{~Pa}$ and $S_{w}^{o} \approx 0.05$ when only a very small fraction of the GDL inlet face is exposed to water (SGL 10BA-P). This situation is illustrated in the left and middle panels of Figure 3.

The $P_{c}-S_{w}$ relationship for water injection into SGL10BB is different depending on whether the MPL is found on the inlet face (SGL10BB-I) or the outlet face (SGL10BB-O) of the sample. In the former case, the data are displaced towards higher capillary pressures, as expected since water invasion into the GDL is controlled by the smaller pore sizes of the intervening MPL. In the latter case, the $P_{c}-S_{w}$ relationship in the low capillary pressure range is essentially the same as in test SGL10BA-F, which is expected since water is first invading the substrate material in both cases. In either case (SGL10BB-I or SGL10BB-O), the maximum water saturation achieved is about $75 \%$, demonstrating that the MPL is not significantly filled with water at the maximum capillary pressure established. When the MPL is on the outlet face, water breakthrough in SGL10BB occurs at $P_{c}^{\circ} \approx 6700 \mathrm{~Pa}$ and $S_{w}^{o} \approx 0.57$. Such high water saturation is easily understood since the GDL is filled substantially by water at the capillary pressure necessary for water to invade the smaller MPL pores. Water 
breakthrough in sample SGL10BB when the MPL is on the inlet face occurs at a similarly high capillary pressure $\left(P_{c}^{\circ} \approx 6000 \mathrm{~Pa}\right)$, but in this case $S_{w}^{o} \approx 0.03$.

The fact that very similar breakthrough saturation is observed in tests SGL 10BA-P and SGL10BB-I supports the conclusion that the MPL limits water access to the GDL face. Water breaks through the MPL at a few isolated locations at the MPL-GDL interface, in the same way water droplets emerge from the GDL at the GDL-gas channel interface $[2,15,16]$, regardless of whether or not the MPL is cracked. Subsequent water percolation through the GDL starts from these locations, as opposed to the entire face of the GDL, as depicted in Figure 3(right). This renders most pores on the GDL face inaccessible to water, dramatically reducing GDL saturation at breakthrough by reducing the number of dead end clusters (a finite-size effect). In this manner, reduced gas diffusivity is confined to a smaller part of the transport domain (the MPL), resulting in improved performance at high current densities.

The breakthrough pressures observed in tests SGL10BA-P and SGL10BB-I should not be expected to be the same due to the more hydrophobic nature and smaller pores of the MPL compared to the hole punctured in the PTFE mask. Furthermore, breakthrough pressures of the order of $6000 \mathrm{~Pa}$, observed in tests SGL10BB-land SGL10BB-O are lower than expected from the pore sizes of the MPL $(<1 \mu \mathrm{m})$. Rather, a breakthrough pressure in this range indicates that cracks and defects in the MPL are the main pathways for water invasion. Interestingly, flow through the MPL via cracks would be beneficial since it eliminates the formation of dead-end clusters in the MPL that are characteristic of percolation processes and contribute significantly to water saturation at breakthrough when finite-size effects are important. In fact, it might be advantageous to design the MPL with strategically placed holes. 


\section{Conclusions}

An explanation for the efficacy of the MPL in fuel cell operation is proposed based on finitesize effects related to invasion percolation in thin GDLs. Water percolation through the MPL results in limited access of water to the GDL inlet face. Consequently, far fewer dead-end water clusters come into being at breakthrough and therefore much lower water saturation is reached. Experimental evidence is provided by a modified version of a recently reported capillary pressure measurement technique [11] that allows both the pressure and saturation at breakthrough to be simultaneously identified. The saturation at breakthrough drops from $27 \%$ to less than $3 \%$ when an MPL intervenes between the GDL and the water source. A similar

result is obtained when an otherwise impermeable mask with a small puncture is placed between the water and the GDL. These findings suggest that the effectiveness of the MPL might be further improved by designing them with large holes for water passage into the GDL, while leaving the micro-porosity dry for gas transport. 


\section{References}

[1] R.Lenormand, C.Zarcone, A.Sarr, Journal of Fluid Mechanics 135 (1983) 337.

[2] P.K.Sinha, C.Y.Wang, Electrochim Acta 52 (2007) 7936.

[3] R.P.Ramasamy, E.C.Kumbur, M.M.Mench, W.Liu, D.Moore, M.Murthy, Int J Hydrogen Energy 33 (2008) 3351.

[4] U.Pasaogullari, C.Y.Wang, J Electrochem Soc 151 (2004) A399.

[5] J.H.Nam, M.Kaviany, Int J Heat Mass Trans 46 (2003) 4595.

[6] A.Z.Weber, J.Newman, J Electrochem Soc 152 (2005) A677.

[7] U.Pasaogullari, C.Y.Wang, K.S.Chen, J Electrochem Soc 152 (2005) A1574.

[8] D.Spernjak, A.K.Prasad, S.G.Advani, J Power Sources 170 (2007) 334.

[9] S.Ge, C.Y.Wang, J Electrochem Soc 154 (2007) B998.

[10] H.K.Atiyeh, K.Karan, B.Peppley, A.Phoenix, E.Halliop, J.Pharoah, J Power Sources 170 (2007) 111.

[11] J.T.Gostick, M.A.Ioannidis, M.W.Fowler, M.D.Pritzker, Electrochem Comm 10 (2008) 1520.

[12] J.Benziger, J.Nehlsen, D.Blackwell, T.Brennan, J.Itescu, J Membrane Sci 261 (2005) 98.

[13] J.T.Gostick, M.W.Fowler, M.A.loannidis, M.D.Pritzker, Y.M.Volfkovich, A.Sakars, J Power Sources 156 (2006) 375.

[14] J.T.Gostick, M.A.Ioannidis, M.W.Fowler, M.D.Pritzker, J Power Sources 173 (2007) 277.

[15] S.Litster, D.Sinton, N.Djilali, J Power Sources 154 (2006) 95.

[16] X.G.Yang, F.Y.Zhang, A.L.Lubawy, C.Y.Wang, Electrochem and Solid-State Let 7 (2004) A408. 


\section{Figure Captions}

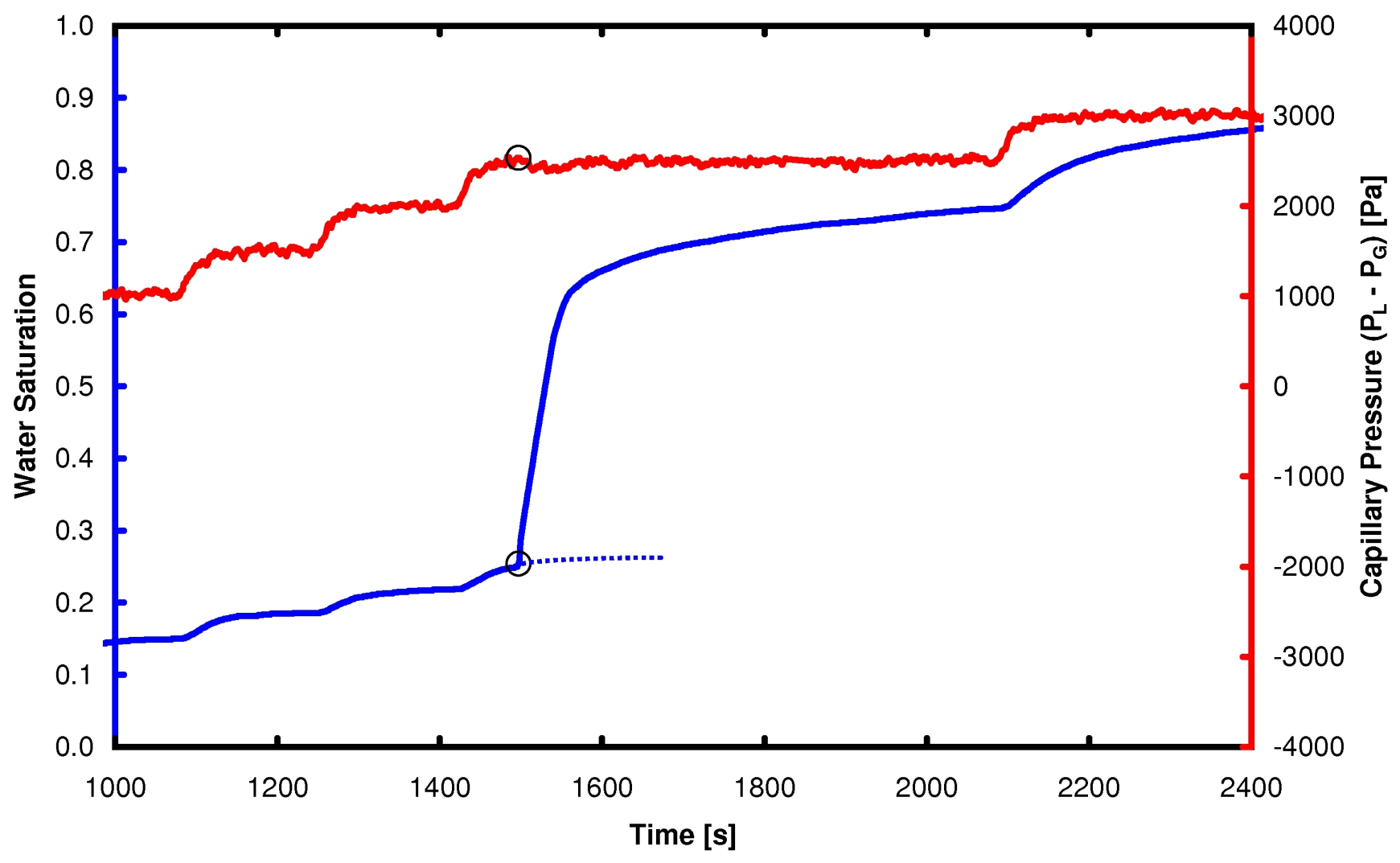

Figure 1: Experimental results from water breakthrough test. Left: Pressure and saturation time traces for SGL10BA showing the breakthrough point. Right: Comparison of capillary pressure curves obtained using original and modified method up to the breakthrough point. 


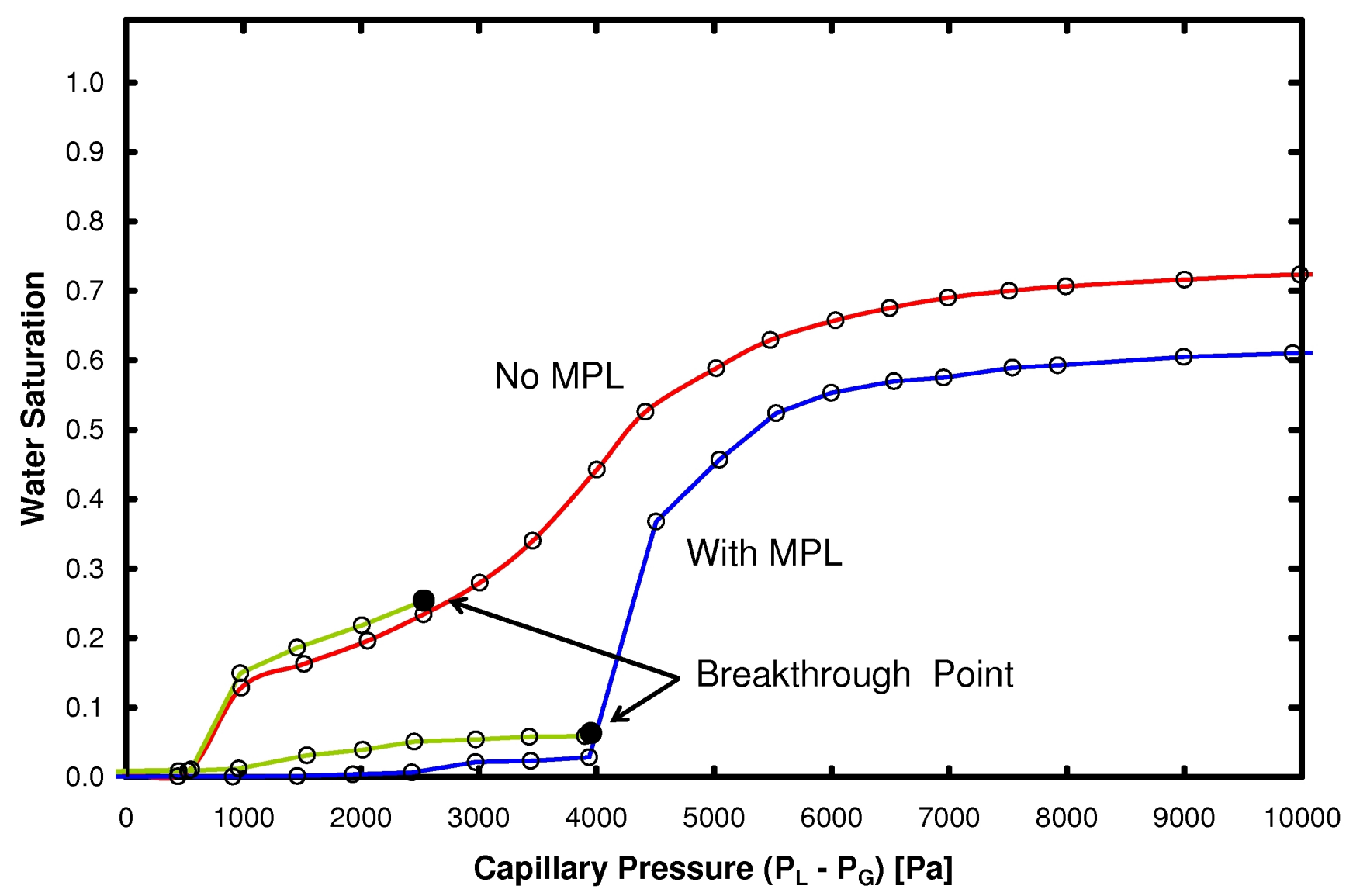

Figure 2: Capillary pressure curves for SGL10BA and SGL10BB with breakthrough points overlaid as filled markers with corresponding shapes. F: Full face exposed to water, P: Punctured mask between water and GDL, I: MPL facing water inlet, O: MPL facing away from water inlet. 

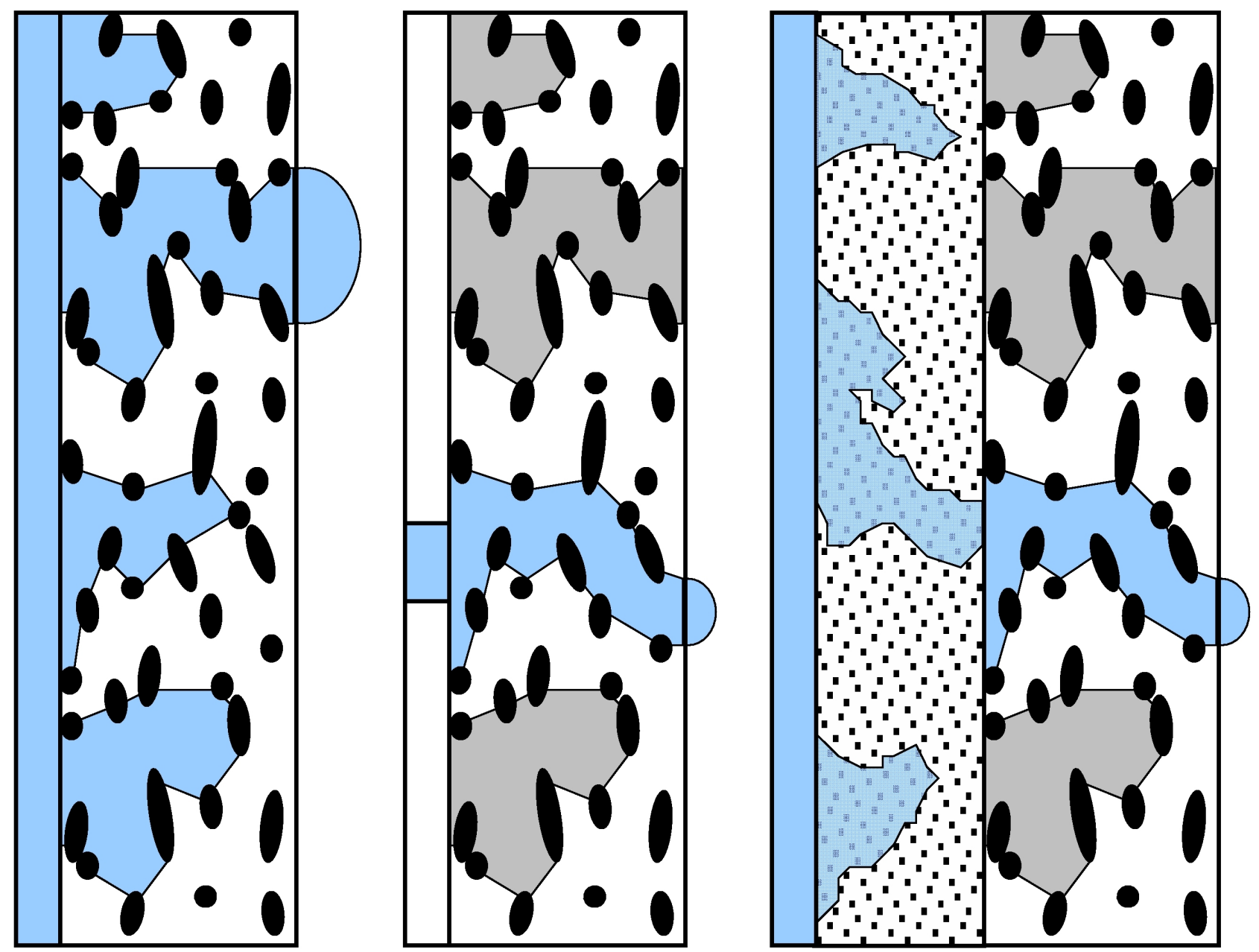

Figure 3: Proposed configuration of water in the GDL with different injection conditions. Left: Full face injection leads to several dead-end clusters and a single breakthrough cluster. Middle: Point source injection leads to a single breakthrough cluster. Right: Percolation through the MPL creates conditions similar to point-like injection into the GDL. Grey shows water and dashed line marks regions that would be filled if they were accessible. 